\title{
Determining Efficacy of Monosodium Glutamate for Salt Reduction in Plain and Spiced 'Poories' Through Sensory Responses
}

Maheshwari HM, Prabhavathi SN, Rajesh Devisetti and Jamuna Prakash*

Department of Food Science and Nutrition, University of Mysore, Manasagangotri, Mysuru-570006, India

\begin{abstract}
Palatability is considered as a major determinant of food selection and intake. Umami foods are being employed widely in savory food formulation for its greater potential for enhancing savory taste. In the present investigation, the role of monosodium glutamate (MSG) in salt reduction along with spices was investigated to understand the flavor potentiating effect and synergistic action of MSG. Deep fried unleavened wheat based traditional Indian bread (Poories) was chosen to study the acceptability. Control product was prepared with $2.0 \%$ salt. For experimental products, two levels of MSG viz, 75 and $100 \mathrm{mg} / 100 \mathrm{~g}$ and three levels of salt, namely, $1.5,1.75$ and $2.0 \%$ were selected. Spices, namely, chili (Capsicum annum L.) cumin (Cuminum cyminum)), pepper (Piper nigrum L) and omum (Trachyspermum ammi)) were incorporated into the product, either as single spice or in combination. The results revealed that the MSG added products were given a higher score for umami flavour in comparison to the control product. In general, MSG added products scored better for flavor in salt reduced products. Results of low sodium poories with single spices and different level of salt indicated that all products prepared with spices were equally liked for the selected sensory characteristics. The products prepared with $1.75 \%$ salt obtained highest scores. This is indicative of the flavour enhancing property of MSG. Similarly for products with mixture of spices; salt reduced products were also accepted well. PCA plot revealed the flavor potentiating effect of MSG in salt reduction. Thus it could be said that using MSG and spices combination would be a better option for achieving salt reduction in savory products.
\end{abstract}

Keywords: Flavour potentiator; Umami; Salt reduction; Sensory attributes; Free choice profiling; Spices

\section{Introduction}

Taste is recognized as one of the most important determining factor for food selection, and this fact is well utilized by food industry to introduce new products in the market. Taste becomes as overriding factor governing and influencing the purchase of food products by consumers. This is followed by other attributes which could be texture, aroma, or the appearance factors [1]. The present-day consumers look for products with dual quality of providing acceptable flavors and promoting health and food manufacturers have to find ways and means of satisfying this particular demand.

Monosodium glutamate (MSG) has long been used as a flavour potentiator [2]. The taste of MSG has been recognized and accepted as a basic taste quality along with others, namely, salt, sweet, sour and bitter [2-4]. Flavour is a combination of multiple attributes of taste, aroma and texture. MSG is known to enhance the existing flavour of products and also has a savoury taste, which contributes to saltiness $[3,4]$.

The flavour potentiating property of MSG has been evaluated in multiple products, all with positive results to indicate that MSG does enhance the natural flavour of foods and increases the level of perceived saltiness $[5,6]$. MSG has low sodium content in comparison to salt and thus can reduce the amount of added salt. It has been demonstrated through studies that adding some MSG to soups and other types of food helps in reducing the amount of added salt [7-9]. Though the taste of MSG is new to Indian palate and it is not used in traditional cuisine, our earlier studies have demonstrated acceptance of MSG in Indian traditional products as well as its efficacy in salt reduction in many different products [10-14].

Sodium chloride or salt is an essential element for all living beings in small quantities. Apart from imparting the necessary flavour, it also controls food properties and is used for its preserving action. However, in recent years its adverse role for human health, if taken in excess is being emphasized with many new epidemiological evidences in different populations. A high salt intake is associated with hypertension and is detrimental to health [1518]. In Indian scenario, fried snacks contribute substantially towards a high salt intake as they carry around $1.8-2.5 \%$ salt [19]. Hence, it is advisable to reduce salt in diet as well as in processed foods to lower the sodium intake. Since salt is an adaptive taste, a certain level of salt is desired for optimum satisfaction. A low salt level can lower the acceptability of product. In this context MSG can act as a flavour potentiator and increase the feeling of saltiness in the food. The present experiment was designed to study this aspect of MSG, wherein the acceptability profile of salt reduced products was investigated at two levels of MSG, namely, $75 \mathrm{mg}$ and $100 \mathrm{mg} / 100 \mathrm{~g}$ of base flour. The product selected for the study was 'Poori'. Poori is deep fried, unleavened flat bread prepared with wheat flour and/or semolina. It is a popular staple dish eaten with a meal or as a snack. Apart from salt reduction, the role of spices was also studied to understand if MSG has a synergistic action with spices in salt reduction.

\section{Materials and Methods}

\section{Materials}

The ingredients used for the preparation of poori were; refined

${ }^{*}$ Corresponding author: Prof. Jamuna Prakash, Department of Food Science and Nutrition, University of Mysore, Manasagangotri, Mysuru-570 006, India, Tel: +91821-2419634; E-mail: jampr55@hotmail.com

Received: July 01, 2017; Accepted: July 27, 2017; Published: August 05, 2017

Citation: Maheshwari HM, Prabhavathi SN, Devisetti R, Prakash J (2017) Determining Efficacy of Monosodium Glutamate for Salt Reduction in Plain and Spiced 'Poories' Through Sensory Responses. J Exp Food Chem 3: 129. doi:10.4172/2472-0542.1000129

Copyright: @ 2017 Maheshwari HM, et al. This is an open-access article distributed under the terms of the Creative Commons Attribution License, which permits unrestricted use, distribution, and reproduction in any medium, provided the original author and source are credited. 
Citation: Maheshwari HM, Prabhavathi SN, Devisetti R, Prakash J (2017) Determining Efficacy of Monosodium Glutamate for Salt Reduction in Plain and Spiced 'Poories' Through Sensory Responses. J Exp Food Chem 3: 129. doi:10.4172/2472-0542.1000129

Page 2 of 9

\begin{tabular}{|c|c|c|c|c|c|c|}
\hline \multirow{2}{*}{ Sensory Attributes } & \multicolumn{4}{|c|}{ Salt Level (\%) } & \multicolumn{2}{|c|}{ ANOVA } \\
\hline & 2.0 (Con.) & 1.5 & 1.75 & 2.0 & F-value & P-value \\
\hline \multicolumn{7}{|c|}{ MSG Level 1 (75 mg/100 g) } \\
\hline Appearance & $8.33 \pm 1.047$ & $8.58 \pm 1.106$ & $8.40 \pm 0.779$ & $8.78 \pm 0.861$ & 1.750 & $0.158^{\text {ns }}$ \\
\hline Texture & $8.63 \pm 1.254$ & $8.93 \pm 0.997$ & $8.68 \pm 0.797$ & $8.70 \pm 0.790$ & 0.737 & $0.530^{\text {ns }}$ \\
\hline Umami flavour & - & $7.63 \pm 1.352^{\mathrm{b}}$ & $8.40 \pm 0.671^{\mathrm{a}}$ & $8.98 \pm 0.729^{a}$ & 12.641 & $0.000^{*+*}$ \\
\hline Overall quality & $8.5 \pm 1.132^{\mathrm{b}}$ & $9.07 \pm 0.916^{a}$ & $9.15 \pm 0.699^{a}$ & $9.25 \pm 0.750^{\mathrm{a}}$ & 5.941 & $0.0007^{* * *}$ \\
\hline \multicolumn{7}{|c|}{ MSG Level $2(100 \mathrm{mg} / 100 \mathrm{~g})$} \\
\hline Appearance & $9.08 \pm 0.729^{a}$ & $9.05 \pm 0.749^{a}$ & $8.40 \pm 0.778^{b}$ & $8.77 \pm 0.862^{a}$ & 6.4692 & $0.0003^{* * *}$ \\
\hline Texture & $8.33 \pm 0.656$ & $8.50 \pm 0.877$ & $8.68 \pm 0.797$ & $8.70 \pm 0.791$ & 1.9781 & $0.1195 \mathrm{~ns}$ \\
\hline Umami flavour & - & $8.25 \pm 0.742^{b}$ & $8.40 \pm 0.672^{b}$ & $8.68 \pm 0.729^{a}$ & 3.6313 & $0.0295^{*}$ \\
\hline Overall quality & $8.98 \pm 0.619$ & $9.13 \pm 0.607$ & $9.15 \pm 0.699$ & $9.28 \pm 0.751$ & 1.3491 & $0.2625 \mathrm{~ns}$ \\
\hline
\end{tabular}

Table 1: Mean sensory scores of Poori (Control) with different levels of Salt and MSG (Note: Different superscripts in rows indicate significant differences among samples on application of DMRT)

wheat flour (Aestivum sativum) and fine wheat semolina, sunflower oil (Helianthus annuus), salt and spices namely, chili (Capsicum annum L.), cumin seeds (Cuminum cyminum), omum (Trachyspermum ammi) and pepper (Piper nigrum L.). These conventionally grown ingredients were procured from super market. Spices were powdered and used in product preparation. Monosodium glutamate used as the flavor enhancing agent was sourced from Ajinomoto company.

\section{Study design}

The study design consisted of preparation of products using single spices or combination of spices with different levels of salt. The control products were prepared using $2 \mathrm{~g}$ of salt per $100 \mathrm{~g}$ flour with the respective spice combinations. All products were prepared using two levels of MSG, $75 \mathrm{mg}$ and $100 \mathrm{mg} / 100 \mathrm{~g}$ of flour including control products. These were subjected to sensory analysis using two types of sensory tests. The data were analyzed using suitable statistical tests.

\section{Preparation of products}

The control product was prepared using $50 \mathrm{~g}$ of refined flour, 50 $\mathrm{g}$ of semolina, $2.0 \mathrm{~g}$ of salt and $10 \mathrm{ml}$ of heated oil for shortening. All the ingredients were mixed well for uniform distribution and soft dough was made by adding appropriate amount of water. The prepared dough was kept aside for half an hour. The dough was divided into small balls of equal size and was rolled into thin round sheets and was deep fried in sunflower oil until done. The frying temperature was $170-175^{\circ} \mathrm{C}$.

The spiced variations were prepared by adding either single spices namely, chili powder $(1.0 \%)$, omum $(0.5 \%)$, pepper $(0.4 \%)$ and cumin seeds $(0.5 \%)$ to the above dough or with two different spice powders as follows: (1) chili+cumin, (2) chili+omum, (3) pepper+cumin and (4) pepper+omum. Cumin seeds were roasted and powdered prior to adding to the dough. Pepper and omum were also crushed coarsely and added while making the dough. Experimental variations were prepared with three levels of salt, 1.5, 1.75 and $2.0 \%$. Two different set of products were prepared with either 50 or $100 \mathrm{mg}$ MSG/100 g of flour.

\section{Sensory analysis}

All products were subjected to sensory analysis using semi-trained panel of 40 members. The panel members familiar with the sensory analysis technique were initially screened for their ability to judge the samples. The quality attributes tested were taste, texture, perception of spices and umami flavor, and overall quality. Responses were also collected for mouth coating property and lingering taste using a graded score card [20]. This was followed by free choice profiling [21] for descriptive quality using a tool which does not require training of panelists. At any point of time, only one set of products was given for analysis to rule out any fatigue among the panelists.

\section{Statistical analysis}

The sensory analysis data were subjected to statistical analysis using mean, standard deviation and analysis of variance and a posttest, Duncan's multiple range test, (DMRT) at $5 \%$ level of probability to determine significant differences in sensory attributes of samples, if any. Further a Principle component analysis plot was also drawn to locate the sensory preferences of low salt products.

\section{Results and Discussion}

The results of study are presented in Tables 1-7 and Figure 1.

\section{Sensory response for control products}

The control products were prepared without the addition of any spice. Table 1 gives the data on sensory profile of products with different levels of salt and two levels of MSG with no added spice. For MSG Level 1 , in general all products were acceptable for appearance and texture. The MSG added products were given a higher score for umami flavour, with

\begin{tabular}{|c|c|c|c|c|c|c|c|c|}
\hline \multirow{2}{*}{\begin{tabular}{|l} 
Sensory \\
Attributes \\
Level of salt (\%) \\
\end{tabular}} & \multirow{2}{*}{$\begin{array}{c}\begin{array}{c}\text { Control } \\
\text { (No MSG) }\end{array} \\
2\end{array}$} & \multicolumn{3}{|c|}{$\begin{array}{l}\text { MSG Level } 1 \\
(75 \mathrm{mg} / 100 \mathrm{~g})\end{array}$} & \multicolumn{4}{|c|}{$\begin{array}{c}\text { MSG Level } 2 \\
(100 \mathrm{mg} / 100 \mathrm{~g})\end{array}$} \\
\hline & & 1.5 & 1.75 & 2 & 2 & 1.5 & 1.75 & 2 \\
\hline \multicolumn{9}{|l|}{ Appearance } \\
\hline Appealing & 40 & 40 & 40 & 40 & 30 & 25 & 20 & 40 \\
\hline Attractive & - & - & - & - & 10 & 15 & 20 & - \\
\hline \multicolumn{9}{|l|}{ Texture } \\
\hline Crunchy & 40 & 40 & 40 & 40 & 40 & 40 & 40 & 40 \\
\hline \multicolumn{9}{|l|}{ Umami flavour } \\
\hline Delightful & - & 35 & 35 & 35 & - & 20 & 29 & 30 \\
\hline Not perceptible & - & 5 & 5 & 5 & - & 11 & 11 & 10 \\
\hline \multicolumn{9}{|l|}{ Overall quality } \\
\hline Delicious & 20 & 20 & 30 & 30 & 30 & 35 & 30 & 20 \\
\hline Pleasant & 20 & 20 & 10 & 10 & 10 & 5 & 10 & 20 \\
\hline \multicolumn{9}{|l|}{ Lingering taste } \\
\hline Yes & 25 & 20 & 20 & 25 & 25 & 30 & 29 & 25 \\
\hline No & 15 & 20 & 20 & 15 & 15 & 10 & 11 & 15 \\
\hline \multicolumn{9}{|l|}{ Mouth coating } \\
\hline Yes & - & 40 & 40 & 40 & - & 40 & 40 & 40 \\
\hline No & 40 & - & - & - & 40 & - & - & - \\
\hline
\end{tabular}

Note: There were no responses for the quality of 'Not appealing' under Appearance; 'Brittle' and 'Hard' under Texture; 'Strong' under Umami flavour and 'Unpleasant' under Overall quality.

Table 2: Free choice profiling of poori (control) with different levels of salt and MSG (number of responses). 
Citation: Maheshwari HM, Prabhavathi SN, Devisetti R, Prakash J (2017) Determining Efficacy of Monosodium Glutamate for Salt Reduction in Plain and Spiced 'Poories' Through Sensory Responses. J Exp Food Chem 3: 129. doi:10.4172/2472-0542.1000129

Page 3 of 9

\begin{tabular}{|c|c|c|c|c|c|c|}
\hline \multirow{2}{*}{ Sensory Attributes } & \multicolumn{4}{|c|}{ Level of Salt (\%) } & \multicolumn{2}{|c|}{ ANOVA } \\
\hline & 2.0 (Con.) & 1.5 & 1.75 & 2.0 & F-value & P-value \\
\hline \multicolumn{7}{|c|}{ MSG Level $1(75 \mathrm{mg} / 100 \mathrm{~g})$} \\
\hline \multicolumn{7}{|l|}{ Chili } \\
\hline Appearance & $9.00 \pm 0.679$ & $8.75 \pm 0.707$ & $9.15 \pm 0.863$ & $8.87 \pm 0.822$ & 1.968 & $0.121^{\text {ns }}$ \\
\hline Texture & $8.85 \pm 0.863$ & $9.00 \pm 0.679$ & $9.20 \pm 0.939$ & $9.00 \pm 0.751$ & 1.243 & $0.296^{\text {ns }}$ \\
\hline Spice flavour & $8.80 \pm 0.757$ & $9.02 \pm 0.697$ & $9.10 \pm 0.955$ & $8.77 \pm 0.733$ & 1.672 & $0.176^{\mathrm{ns}}$ \\
\hline Umami flavour & - & $8.68 \pm 0.694^{b}$ & $9.20 \pm 0.822^{\mathrm{a}}$ & $8.77 \pm 0.800^{\mathrm{b}}$ & 5.184 & $0.006^{*}$ \\
\hline Overall quality & $9.30 \pm 0.686$ & $9.35 \pm 0.533$ & $9.45 \pm 0.677$ & $9.30 \pm 0.563$ & 0.521 & $0.668^{\text {ns }}$ \\
\hline \multicolumn{7}{|l|}{ Omum } \\
\hline Appearance & $8.73 \pm 0.678$ & $8.68 \pm 0.693$ & $8.82 \pm 0.384$ & $8.83 \pm 0.384$ & 0.726 & $0.538^{\text {ns }}$ \\
\hline Texture & $8.72 \pm 0.678^{b}$ & $8.55 \pm 0.677^{b}$ & $8.82 \pm 0.501^{\mathrm{b}}$ & $9.20 \pm 0.822^{\mathrm{a}}$ & 6.531 & $0.0003^{*+* *}$ \\
\hline Spice flavour & $8.38 \pm 0.774^{b}$ & $8.58 \pm 0.712^{\mathrm{ab}}$ & $8.53 \pm 0.678^{\mathrm{ab}}$ & $8.63 \pm 0.585^{\mathrm{a}}$ & 0.977 & $0.406^{\mathrm{ns}}$ \\
\hline Umami flavour & - & $8.22 \pm 0.619^{b}$ & $8.35 \pm 0.735^{\mathrm{b}}$ & $8.57 \pm 0.594^{a}$ & 2.953 & $0.056^{\text {ns }}$ \\
\hline Overall quality & $9.03 \pm 0.659 b$ & $9.05 \pm 0.552^{b}$ & $8.83 \pm 0.549^{b}$ & $9.35 \pm 0.769^{\mathrm{a}}$ & 4.589 & $0.0041^{* *}$ \\
\hline \multicolumn{7}{|l|}{ Cumin } \\
\hline Appearance & $9.03 \pm 0.619$ & $9.13 \pm 0.515$ & $9.13 \pm 0.515$ & $9.23 \pm 0.619$ & 0.820 & $0.484^{\text {ns }}$ \\
\hline Texture & $9.13 \pm 0.869$ & $9.08 \pm 0.572$ & $9.23 \pm 0.659$ & $9.88 \pm 0.594$ & 0.420 & $0.738^{\text {ns }}$ \\
\hline Spice flavour & $8.48 \pm 0.505^{b}$ & $8.43 \pm 0.500^{b}$ & $8.83 \pm 0.594^{a}$ & $8.85 \pm 0.735^{\mathrm{a}}$ & 5.777 & $0.0009^{* * *}$ \\
\hline Umami flavour & - & $8.75 \pm 0.500^{b}$ & $8.43 \pm 0.635^{b}$ & $8.85 \pm 0.483^{a}$ & 6.274 & $0.0025^{* *}$ \\
\hline Overall quality & $9.23 \pm 0.530$ & $9.13 \pm 0.334$ & $9.08 \pm 0.474$ & $9.28 \pm 0.554$ & 1.440 & $0.233^{\text {ns }}$ \\
\hline \multicolumn{7}{|l|}{ Pepper } \\
\hline Appearance & $8.78 \pm 0.429^{b}$ & $9.00 \pm 0.0^{\mathrm{a}}$ & $8.83 \pm 0.446^{b}$ & $8.80 \pm 0.464^{b}$ & 2.807 & $0.041^{*}$ \\
\hline Texture & $8.40 \pm 0.296^{b}$ & $8.82 \pm 0.675^{\mathrm{a}}$ & $8.52 \pm 0.554^{b}$ & $8.60 \pm 0.747^{\text {ab }}$ & 3.263 & $0.023^{*}$ \\
\hline Spice flavour & $8.45 \pm 0.503$ & $8.33 \pm 0.615$ & $8.33 \pm 0.655$ & $8.25 \pm 0.630$ & 0.753 & $0.522^{\text {ns }}$ \\
\hline Umami flavour & - & $8.30 \pm 0.464$ & $8.10 \pm 0.545$ & $8.32 \pm 0.655$ & 1.935 & $0.148^{\text {ns }}$ \\
\hline Overall quality & $8.93 \pm 0.266^{\mathrm{bc}}$ & $9.3 \pm 0.464^{a}$ & $8.83 \pm 0.446^{c}$ & $9.05 \pm 0.552^{b}$ & 8.512 & $0.000^{*+*+1}$ \\
\hline \multicolumn{7}{|c|}{ MSG Level $2(100 \mathrm{mg} / 100 \mathrm{~g})$} \\
\hline \multicolumn{7}{|l|}{ Chili } \\
\hline Appearance & $8.95 \pm 0.714$ & $9.15 \pm 0.864$ & $8.87 \pm 0.822$ & $8.75 \pm 0.707$ & 1.8463 & $0.1410^{\text {ns }}$ \\
\hline Texture & $8.80 \pm 0.853$ & $9.20 \pm 0.939$ & $9.00 \pm 0.751$ & $9.00 \pm 0.679$ & 1.6186 & $0.1872^{\text {ns }}$ \\
\hline Spice flavour & $8.75 \pm 0.742$ & $9.10 \pm 0.955$ & $8.78 \pm 0.733$ & $9.03 \pm 0.698$ & 1.9958 & $0.1168^{\text {ns }}$ \\
\hline Umami flavour & - & $9.20 \pm 0.823^{\mathrm{a}}$ & $8.78 \pm 0.800^{\mathrm{b}}$ & $8.68 \pm 0.694^{b}$ & 5.1842 & $0.0069^{* *}$ \\
\hline Overall quality & $9.20 \pm 0.723$ & $9.45 \pm 0.877$ & $9.30 \pm 0.564$ & $9.35 \pm 0.533$ & 1.0938 & $0.3535^{\mathrm{ns}}$ \\
\hline \multicolumn{7}{|l|}{ Omum } \\
\hline Appearance & $8.73 \pm 0.679$ & $8.83 \pm 0.385$ & $8.83 \pm 0.385$ & $9.68 \pm 0.694$ & 0.7267 & $0.5374^{\text {ns }}$ \\
\hline Texture & $8.73 \pm 0.679$ & $8.83 \pm 0.501$ & $9.20 \pm 0.823$ & $8.55 \pm 0.677$ & 6.5315 & $6.5315^{\text {ns }}$ \\
\hline Spice flavour & $8.38 \pm 0.774$ & $8.53 \pm 0.679$ & $8.63 \pm 0.586$ & $8.76 \pm 0.712$ & 0.9771 & $0.4051^{\text {ns }}$ \\
\hline Umami flavour & - & $8.35 \pm 0.736$ & $8.58 \pm 0.594$ & $8.23 \pm 0.619$ & 2.9533 & $0.0560^{\text {ns }}$ \\
\hline Overall quality & $9.03 \pm 0.659 b$ & $8.83 \pm 0.549 b$ & $9.35 \pm 0.769 a$ & $9.05 \pm 0.552 b$ & 4.5882 & $0.0041^{* *}$ \\
\hline \multicolumn{7}{|l|}{ Cumin } \\
\hline Appearance & $9.05 \pm 0.597$ & $9.23 \pm 0.619$ & $9.13 \pm 0.516$ & $9.22 \pm 0.516$ & 0.6483 & $0.5851^{\mathrm{ns}}$ \\
\hline Texture & $9.20 \pm 0.687$ & $9.18 \pm 0.594$ & $9.08 \pm 0.572$ & $9.23 \pm 0.659$ & 0.4356 & $0.7278^{\text {ns }}$ \\
\hline Spice flavour & $8.55 \pm 0.552^{b}$ & $8.85 \pm 0.736^{b}$ & $8.43 \pm 0.501^{a}$ & $8.83 \pm 0.594^{a}$ & 4.0845 & $0.0031^{* *}$ \\
\hline Umami flavour & - & $8.85 \pm 0.483^{a}$ & $8.58 \pm 0.501^{b}$ & $8.43 \pm 0.636^{b}$ & 6.2748 & $0.0025^{* \prime}$ \\
\hline Overall quality & $9.3 \pm 0.5638$ & $9.27 \pm 0.554$ & $9.13 \pm 0.335$ & $9.07 \pm 0.474$ & 2.0353 & $0.1111^{\mathrm{ns}}$ \\
\hline \multicolumn{7}{|l|}{ Pepper } \\
\hline Appearance & $8.78 \pm 0.423^{b}$ & $9.00 \pm 0.0^{a}$ & $8.80 \pm 0.464^{b}$ & $8.83 \pm 0.446^{b}$ & 2.8077 & $0.0414^{*}$ \\
\hline Texture & $8.4 \pm 0.496^{b}$ & $8.80 \pm 0.687^{a}$ & $8.60 \pm 0.744^{\mathrm{ab}}$ & $8.53 \pm 0.554^{\mathrm{ab}}$ & 2.8449 & $0.0395^{*}$ \\
\hline Spice flavour & $8.45 \pm 0.504$ & $8.34 \pm 0.622$ & $8.25 \pm 0.630$ & $8.30 \pm 0.687$ & 0.7724 & $0.5110^{\mathrm{ns}}$ \\
\hline Umami flavour & - & $8.30 \pm .4640^{\mathrm{a}}$ & $8.30 \pm 0.648$ & $8.10 \pm 0.545$ & 1.7142 & $0.1845^{\mathrm{ns}}$ \\
\hline Overall quality & $8.925 \pm 0.26^{\mathrm{bc}}$ & $9.33 \pm 0.474^{a}$ & $9.05 \pm 0.552^{b}$ & $8.83 \pm 0.446^{c}$ & 9.357 & $0.000^{* * *}$ \\
\hline
\end{tabular}

Table 3: Mean sensory scores of poori with single spice and different levels of salt and MSG (Note: Different superscripts in rows indicate significant differences among samples on application of DMRT).

the scores showing an increase with increasing levels of salt. The overall quality also showed a significant increase in scores with added MSG when compared with control product and did not show any significant difference between different salt levels. This data indicates that MSG increased the taste profile of poories with different salt levels. The poories were acceptable even with salt reduction to $1.5 \%$ and $1.75 \%$. (The control product was prepared with $2.0 \%$ salt). The poories were also prepared with different levels of salt and a higher level of MSG at $100 \mathrm{mg}$. This was 
Citation: Maheshwari HM, Prabhavathi SN, Devisetti R, Prakash J (2017) Determining Efficacy of Monosodium Glutamate for Salt Reduction in Plain and Spiced 'Poories' Through Sensory Responses. J Exp Food Chem 3: 129. doi:10.4172/2472-0542.1000129

Page 4 of 9

\begin{tabular}{|c|c|c|c|c|c|c|c|c|c|c|c|c|c|c|c|c|}
\hline \multirow[b]{2}{*}{ Sensory Attributes } & \multicolumn{16}{|c|}{ Salt level (\%) } \\
\hline & $\begin{array}{l}\dot{\text { j}} \\
\dot{\delta} \\
\dot{\delta}\end{array}$ & $\stackrel{n}{\sim}$ & $\stackrel{\mathfrak{R}}{\stackrel{2}{r}}$ & $\stackrel{\circ}{\mathrm{i}}$ & $\begin{array}{l}\stackrel{0}{\mathrm{j}} \\
\dot{\delta} \\
\dot{0}\end{array}$ & $\stackrel{n}{\sim}$ & 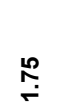 & $\stackrel{\circ}{\text { i }}$ & $\begin{array}{l}\stackrel{\circ}{\mathrm{j}} \\
\dot{\delta} \\
\dot{0}\end{array}$ & $\stackrel{n}{\sim}$ & $\stackrel{\mathfrak{R}}{\stackrel{2}{\sim}}$ & $\stackrel{\circ}{\text { i }}$ & $\begin{array}{l}\stackrel{\circ}{\mathrm{j}} \\
\dot{\delta} \\
\dot{0}\end{array}$ & $\stackrel{n}{\sim}$ & 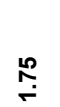 & $\stackrel{\circ}{i}$ \\
\hline Spices & \multicolumn{4}{|c|}{ Chili } & \multicolumn{4}{|c|}{ Omum } & \multicolumn{4}{|c|}{ Cumin } & \multicolumn{4}{|c|}{ Pepper } \\
\hline \multicolumn{17}{|c|}{ MSG Level $1(75 \mathrm{mg} / 100 \mathrm{~g})$} \\
\hline \multicolumn{17}{|l|}{ Appearance } \\
\hline Appealing & 30 & 25 & 40 & 40 & 40 & 40 & 40 & 40 & 30 & 30 & 25 & 30 & 40 & 40 & 40 & 40 \\
\hline Attractive & 10 & 15 & - & - & - & - & - & - & 10 & 10 & 15 & 10 & - & - & - & - \\
\hline \multicolumn{17}{|l|}{ Texture } \\
\hline Crunchy & 40 & 40 & 40 & 40 & 40 & 40 & 40 & 40 & 40 & 40 & 40 & 40 & 40 & 40 & 40 & 40 \\
\hline \multicolumn{17}{|l|}{ Spice flavour } \\
\hline Delightful & - & - & - & - & 40 & 40 & 40 & 40 & - & - & - & - & 40 & 40 & 40 & 40 \\
\hline \multicolumn{17}{|l|}{ Umami flavour } \\
\hline Delightful & - & 35 & 36 & 35 & - & 35 & 35 & 35 & - & 35 & 35 & 35 & - & 40 & 40 & 40 \\
\hline Not perceptible & - & 5 & 4 & 5 & - & 5 & 5 & 5 & - & 5 & 5 & 5 & - & - & - & - \\
\hline \multicolumn{17}{|l|}{ Overall quality } \\
\hline Delicious & 30 & 30 & 30 & 30 & 30 & 30 & 40 & 40 & 30 & 30 & 40 & 40 & 20 & 25 & 30 & 30 \\
\hline Pleasant & 10 & 10 & 10 & 10 & 10 & 10 & - & - & 10 & 10 & - & - & 20 & 15 & 10 & 10 \\
\hline \multicolumn{17}{|l|}{ Lingering taste } \\
\hline Yes & 30 & 30 & 40 & 35 & 25 & 30 & 40 & 35 & 25 & 30 & 40 & 35 & 40 & 40 & 40 & 40 \\
\hline No & 10 & 10 & - & 5 & 15 & 10 & - & 5 & 15 & 10 & - & 5 & - & - & - & - \\
\hline \multicolumn{17}{|l|}{ Mouth coating } \\
\hline Yes & - & 40 & 40 & 40 & - & 40 & 40 & 40 & - & 40 & 40 & 40 & - & 40 & 40 & 40 \\
\hline No & 40 & - & - & - & 40 & - & - & & 40 & - & - & - & 40 & - & - & - \\
\hline \multicolumn{17}{|c|}{ MSG Level $2(100 \mathrm{mg} / 100 \mathrm{~g})$} \\
\hline \multicolumn{17}{|l|}{ Appearance } \\
\hline Appealing & 35 & 30 & 30 & 40 & 35 & 23 & 25 & 25 & 35 & 30 & 30 & 35 & 40 & 30 & 40 & 31 \\
\hline Attractive & 5 & 10 & 10 & & 5 & 17 & 15 & 15 & 5 & 10 & 10 & 5 & - & 10 & - & 9 \\
\hline \multicolumn{17}{|l|}{ Texture } \\
\hline Crunchy & 40 & 40 & 40 & 40 & 40 & 40 & 40 & 40 & 40 & 40 & 40 & 40 & 40 & 40 & 40 & 40 \\
\hline \multicolumn{17}{|l|}{ Spice flavour } \\
\hline Delightful & 40 & 40 & 40 & 40 & 40 & 40 & 40 & 40 & 40 & 40 & 40 & 40 & 40 & 40 & 40 & 40 \\
\hline \multicolumn{17}{|l|}{ Umami flavour } \\
\hline Delightful & - & 35 & 35 & 35 & - & 35 & 35 & 35 & - & 40 & 40 & 40 & & 35 & 35 & 40 \\
\hline Not perceptible & - & 5 & 5 & 5 & - & 5 & 5 & 5 & - & - & - & - & - & 5 & 5 & - \\
\hline Overall quality & & & & & & & & & & & & & & & & \\
\hline Delicious & 25 & 30 & 29 & 25 & 25 & 30 & 29 & 25 & 25 & 30 & 30 & 30 & 25 & 30 & 29 & 25 \\
\hline Pleasant & 15 & 10 & 11 & 15 & 15 & 10 & 11 & 15 & 15 & 10 & 10 & 10 & 15 & 10 & 11 & 15 \\
\hline Lingering taste & & & & & & & & & & & & & & & & \\
\hline Yes & 32 & 40 & 40 & 40 & 30 & 40 & 40 & 40 & 35 & 40 & 40 & 40 & 40 & 40 & 40 & 40 \\
\hline No & 8 & - & - & - & 10 & & - & - & 5 & - & - & - & - & - & - & - \\
\hline Mouth coating & & & & & & & & & & & & & & & & \\
\hline Yes & - & 40 & 40 & 40 & - & 40 & 40 & 40 & - & 40 & 40 & 40 & - & 40 & 40 & 40 \\
\hline No & 40 & - & - & - & 40 & - & - & - & 40 & - & - & - & 40 & - & - & - \\
\hline
\end{tabular}

No response for 'not appealing' under Appearance; 'brittle' and 'hard' under Texture; 'strong' and 'not perceptible' under Spice flavour; 'strong' under Umami flavour; and 'unpleasant' under Overall quality.

Table 4: Free choice profiling of poori with single spices and different levels of salt and MSG (number of responses).

designed to bring out the flavour potentiating effect of MSG at a higher level using same levels of salt. Here significant differences were observed for appearance and umami flavour. While the response for appearance cannot be explained, the umami flavour enhanced with a higher level of salt. All other attributes were similar. Products with lower level of salt were also given higher scores for overall quality indicating that MSG enhanced the salty flavour of such products.

For obtaining enhanced salty taste, flavour enhancing natural ingredients could be employed in food formulation which helps to obtain savory profile and rebalance the flavor of products containing low sodium along with contribution of increased saltiness perception [22]. Since salt reduction is known to have greater impact on flavour perception, an investigation on the flavour and textural properties of white bread containing $10-50 \%$ less sodium in which salt was replaced with fermented soy sauce at 25,50 and $100 \%$ was conducted. The study showed that there was an increased perception of salty taste. This suggested that salt could be effectively reduced up to $40 \%$ by replacing it with a natural flavour enhancing ingredient. Salt replacement with 
Citation: Maheshwari HM, Prabhavathi SN, Devisetti R, Prakash J (2017) Determining Efficacy of Monosodium Glutamate for Salt Reduction in Plain and Spiced 'Poories' Through Sensory Responses. J Exp Food Chem 3: 129. doi:10.4172/2472-0542.1000129

Page 5 of 9

\begin{tabular}{|c|c|c|c|c|c|c|}
\hline \multirow{2}{*}{ Sensory Attributes } & \multicolumn{4}{|c|}{ Level of Salt } & \multicolumn{2}{|c|}{ ANOVA } \\
\hline & 2.0 (Con.) & 1.5 & 1.75 & 2.0 & F-value & P-value \\
\hline \multicolumn{7}{|c|}{ MSG Level 1 (75 mg/100 g) } \\
\hline \multicolumn{7}{|l|}{ Chili+Omum } \\
\hline Appearance & $9.22 \pm 0.768$ & $9.45 \pm 0.714$ & $9.38 \pm 0.807$ & $9.43 \pm 0.781$ & 0.6886 & $0.5602^{\text {ns }}$ \\
\hline Texture & $8.78 \pm 0.733^{b}$ & $9.35 \pm 0.622^{a}$ & $9.30 \pm 0.823^{a}$ & $9.45 \pm 0.597^{a}$ & 7.2775 & $0.0001^{* * *}$ \\
\hline Spice flavour & $8.75 \pm 0.494^{b}$ & $8.90 \pm 0.632^{b}$ & $9.02 \pm 0.920^{\mathrm{ab}}$ & $9.25 \pm 0.776^{a}$ & 3.4223 & $0.0187^{\star}$ \\
\hline Umami flavour & - & $8.58 \pm 0.501^{b}$ & $8.63 \pm 0.705^{b}$ & $9.10 \pm 0.841^{\mathrm{a}}$ & 6.9237 & $0.0014^{\star *}$ \\
\hline Overall quality & $9.13 \pm 0.463$ & $9.30 \pm 0.516$ & $9.30 \pm 0.723$ & $9.32 \pm 0.616$ & 0.9879 & $0.4001^{\text {ns }}$ \\
\hline \multicolumn{7}{|l|}{ Chili+Cumin } \\
\hline Appearance & $8.75 \pm 0.707^{b}$ & $8.30 \pm 0.464^{a}$ & $9.20 \pm 0.405^{a}$ & $9.30 \pm 0.464^{a}$ & 10.077 & $0.000^{* * *}$ \\
\hline Texture & $8.50 \pm 0.677$ & $8.80 \pm 0.405$ & $8.80 \pm 0.758$ & $8.80 \pm 0.405$ & 1.836 & $0.1428^{\text {ns }}$ \\
\hline Spice flavour & $8.30 \pm 0.911^{\mathrm{ab}}$ & $8.00 \pm 0.405^{b}$ & $8.60 \pm 0.496^{a}$ & $8.00 \pm 0.641^{\mathrm{b}}$ & 6.957 & $0.0002^{* * *}$ \\
\hline Umami flavour & - & $8.50 \pm 0.679^{b}$ & $8.80 \pm 0.405^{a}$ & $8.50 \pm 0.679^{b}$ & 3.311 & $0.0399^{*}$ \\
\hline Overall quality & $8.90 \pm 0.545^{b}$ & $9.20 \pm 0.405^{a}$ & $9.30 \pm 0.464^{a}$ & $9.20 \pm 0.405^{a}$ & 5.707 & $0.0009^{* * *}$ \\
\hline \multicolumn{7}{|l|}{ Pepper+Omum } \\
\hline Appearance & $9.00 \pm 0.641^{b}$ & $9.20 \pm 0.405^{\mathrm{ab}}$ & $9.00 \pm 0.641^{b}$ & $9.30 \pm 0.464^{a}$ & 3.0 & $0.0323^{*}$ \\
\hline Texture & $8.80 \pm 0.405$ & $8.80 \pm 0.758$ & $8.60 \pm 0.496$ & $8.80 \pm 0.405$ & 1.393 & $0.2470^{\text {ns }}$ \\
\hline Spice flavour & $8.60 \pm 0.496^{a}$ & $8.60 \pm 0.496^{a}$ & $8.20 \pm 0.758^{b}$ & $8.00 \pm 0.641^{\mathrm{b}}$ & 9.75 & $0.0000^{* * *}$ \\
\hline Umami flavour & - & $8.80 \pm 0.405^{a}$ & $8.20 \pm 0.608^{c}$ & $8.50 \pm 0.679^{b}$ & 10.856 & $0.0000^{* * *}$ \\
\hline Overall quality & $9.10 \pm 0.545$ & $9.30 \pm 0.464$ & $9.20 \pm 0.405$ & $9.20 \pm 0.405$ & 1.268 & $0.2872^{\text {ns }}$ \\
\hline \multicolumn{7}{|l|}{ Pepper+Cumin } \\
\hline Appearance & $8.75 \pm 0.707^{b}$ & $9.30 \pm 0.464^{a}$ & $9.20 \pm 0.405^{a}$ & $9.30 \pm 0.464^{a}$ & 10.077 & $0.0000^{* * *}$ \\
\hline Texture & $8.55 \pm 0.677$ & $8.80 \pm 0.405$ & $8.80 \pm 0.758$ & $8.80 \pm 0.405$ & 1.8361 & $0.1428^{\text {ns }}$ \\
\hline Spice flavour & $8.30 \pm 0.911^{\mathrm{ab}}$ & $8.00 \pm 0.641^{b}$ & $8.60 \pm 0.496^{a}$ & $8.00 \pm 0.645^{\mathrm{b}}$ & 6.9567 & $0.0002^{* * *}$ \\
\hline Umami flavour & - & $8.50 \pm 0.679^{b}$ & $8.80 \pm 0.405^{a}$ & $8.50 \pm 0.679^{b}$ & 3.3113 & $0.0399^{*}$ \\
\hline Overall quality & $8.90 \pm 545^{b}$ & $9.20 \pm 0.405^{a}$ & $9.30 \pm 0.464^{a}$ & $9.20 \pm 0.405^{a}$ & 5.7073 & $0.0009^{*+*}$ \\
\hline \multicolumn{7}{|c|}{ MSG Level $2(100 \mathrm{mg} / 100 \mathrm{~g})$} \\
\hline \multicolumn{7}{|l|}{ Chili+Omum } \\
\hline Appearance & $9.23 \pm 0.768$ & $9.43 \pm 0.712$ & $9.43 \pm 0.781$ & $9.38 \pm 0.807$ & 0.6082 & $0.6105^{\text {ns }}$ \\
\hline Texture & $8.75 \pm 0.707^{b}$ & $9.33 \pm 0.656^{a}$ & $9.45 \pm 0.590^{a}$ & $9.30 \pm 0.823^{a}$ & 7.8899 & $0.0000^{* * *}$ \\
\hline Spice flavour & $8.70 \pm 0.516^{b}$ & $8.93 \pm 0.616^{\mathrm{ab}}$ & $9.25 \pm 0.777^{a}$ & $9.03 \pm 0.920^{\mathrm{ab}}$ & 3.9804 & $0.0091^{* *}$ \\
\hline Umami flavour & - & $8.58 \pm 0.50^{\mathrm{b}}$ & $9.10 \pm 0.841^{a}$ & $8.63 \pm 0.705^{b}$ & 6.9237 & $0.0014^{* * *}$ \\
\hline Overall quality & $9.13 \pm 0.463$ & $9.30 \pm 0.516$ & $9.33 \pm 0.616$ & $9.30 \pm 0.723$ & 0.9879 & $0.4001^{\text {ns }}$ \\
\hline \multicolumn{7}{|l|}{ Chili+Cumin } \\
\hline Appearance & $9.00 \pm 0.641$ & $9.10 \pm 0.304$ & $9.00 \pm 0.641$ & $9.20 \pm 0.405$ & 1.3619 & $0.2565^{\text {ns }}$ \\
\hline Texture & $8.80 \pm 0.405$ & $8.58 \pm 0.675$ & $8.60 \pm 0.496$ & $8.80 \pm 0.758$ & 1.6835 & $0.1727^{\mathrm{ns}}$ \\
\hline Spice flavour & $8.60 \pm 0.496^{a}$ & $8.60 \pm 0.496^{a}$ & $8.20 \pm 0.758^{b}$ & $8.60 \pm 0.406^{a}$ & 4.875 & $0.0028^{* *}$ \\
\hline Umami flavour & - & $8.70 \pm 0.464^{a}$ & $8.20 \pm 0.608^{b}$ & $8.60 \pm 0.496^{a}$ & 10.1111 & $0.0000^{* * *}$ \\
\hline Overall quality & $9.10 \pm 0.545$ & $9.15 \pm 0.362$ & $9.20 \pm 0.405$ & $9.30 \pm 0.464$ & 1.4444 & $0.2320^{\text {ns }}$ \\
\hline \multicolumn{7}{|l|}{ Pepper+Omum } \\
\hline Appearance & $8.75 \pm 0.707^{c}$ & $9.10 \pm 0.304^{\mathrm{ab}}$ & $9.30 \pm 0.464^{a}$ & $9.00 \pm 0.641^{b}$ & 6.8694 & $0.0002^{* *+}$ \\
\hline Texture & $8.55 \pm 0.677$ & $8.58 \pm 0.675$ & $8.80 \pm 0.405$ & $8.60 \pm 0.496$ & 1.5786 & $0.1967^{\text {ns }}$ \\
\hline Spice flavour & $8.30 \pm 0.911$ & $8.35 \pm 0.622$ & $8.00 \pm 0.641$ & $8.20 \pm 0.758$ & 1.7403 & $0.1609^{\text {ns }}$ \\
\hline Umami flavour & - & $8.70 \pm 0.464^{a}$ & $8.50 \pm 0.679^{a}$ & $8.20 \pm 0.608^{b}$ & 7.2647 & $0.0010^{*+*}$ \\
\hline Overall quality & $8.90 \pm 0.545^{b}$ & $9.15 \pm 0.362^{\mathrm{a}}$ & $9.20 \pm 0.405^{\mathrm{a}}$ & $9.20 \pm 0.405^{\mathrm{a}}$ & 4.3627 & $0.0055^{* *}$ \\
\hline \multicolumn{7}{|l|}{ Pepper+Cumin } \\
\hline Appearance & $9.00 \pm 0.642$ & $9.10 \pm 0.304$ & $9.00 \pm 0.641$ & $9.20 \pm 0.405$ & 1.3619 & $0.2565^{\text {ns }}$ \\
\hline Texture & $8.8 \pm 0.405$ & $8.58 \pm 0.675$ & $8.60 \pm 0.496$ & $8.80 \pm 0.758$ & 1.6835 & $0.1727^{\mathrm{ns}}$ \\
\hline Spice flavour & $8.60 \pm 0.496^{a}$ & $8.60 \pm 0.496^{a}$ & $8.20 \pm 0.758^{b}$ & $8.60 \pm 0.496^{a}$ & 4.875 & $0.0028^{* *}$ \\
\hline Umami flavour & - & $8.70 \pm 0.464^{a}$ & $8.20 \pm 0.608^{b}$ & $8.60 \pm 0.496^{a}$ & 10.1111 & $0.000^{* * *+}$ \\
\hline Overall quality & $9.10 \pm 0.545$ & $9.15 \pm 0.362$ & $9.20 \pm 0.405$ & $9.30 \pm 0.464$ & 1.4444 & $0.2320^{\text {ns }}$ \\
\hline
\end{tabular}

Table 5: Mean sensory scores of poori with mixture of spices and different levels of salt and MSG.

natural flavour enhancer to the tune of $25 \%$ was found not to affect the overall liking of bread. This could be due to the fact that soy sauce is known to contain distinct taste quality known as umami [23].

The products were also evaluated using free choice profiling and results are compiled in Table 2. The descriptive quality profile of poories with different levels of salt and $75 \mathrm{mg}$ of MSG indicated a uniform response by all the panel members. The products were described as appealing, crunchy, delightful, delicious and pleasant. For the lingering taste around half of them gave a positive response. Mouth coating was expressed only for MSG added products. These results show that low sodium products were equally acceptable when prepared with small amounts of MSG. For products with higher MSG levels, almost similar responses were obtained, though some of the respondents did not perceive the umami flavour that well. This may have happened due 
Citation: Maheshwari HM, Prabhavathi SN, Devisetti R, Prakash J (2017) Determining Efficacy of Monosodium Glutamate for Salt Reduction in Plain and Spiced 'Poories' Through Sensory Responses. J Exp Food Chem 3: 129. doi:10.4172/2472-0542.1000129

Page 6 of 9

\begin{tabular}{|c|c|c|c|c|c|c|c|c|c|c|c|c|c|c|c|c|}
\hline \multirow[b]{2}{*}{ Sensory Attributes } & \multicolumn{16}{|c|}{ Salt level (\%) } \\
\hline & $\begin{array}{l}\stackrel{0}{\text { j }} \\
\dot{\delta} \\
\dot{0}\end{array}$ & $\stackrel{\text { L }}{\leftarrow}$ & 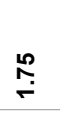 & $\stackrel{\circ}{i}$ & $\begin{array}{l}\stackrel{\text { ì }}{\text { jo }} \\
\dot{\delta}\end{array}$ & $\stackrel{\leftrightarrow}{\leftarrow}$ & $\stackrel{\mathfrak{L}}{\mathfrak{r}}$ & $\stackrel{\text { i }}{ }$ & $\begin{array}{l}\stackrel{i}{\mathrm{~N}} \\
\dot{\delta} \\
\dot{\delta}\end{array}$ & $\stackrel{\leftrightarrow}{\leftarrow}$ & $\stackrel{\stackrel{n}{ }}{=}$ & $\stackrel{\circ}{i}$ & $\begin{array}{l}\stackrel{i}{\text { i }} \\
\dot{\delta} \\
\dot{0}\end{array}$ & $\stackrel{\leftrightarrow}{\leftarrow}$ & $\stackrel{\mathfrak{R}}{\stackrel{2}{\prime}}$ & $\stackrel{\circ}{i}$ \\
\hline Spices & \multicolumn{4}{|c|}{ Chili+Omum } & \multicolumn{4}{|c|}{ Chili+Cumin } & \multicolumn{4}{|c|}{ Pepper+Omum } & \multicolumn{4}{|c|}{ Pepper+Cumin } \\
\hline \multicolumn{17}{|c|}{ MSG Level $1(75 \mathrm{mg} / 100 \mathrm{~g})$} \\
\hline \multicolumn{17}{|l|}{ Appearance } \\
\hline Appealing & 30 & 20 & 15 & 30 & 40 & 40 & 40 & 40 & 30 & 25 & 33 & 26 & 30 & 26 & 28 & 40 \\
\hline Attractive & 10 & 20 & 25 & 10 & - & - & - & - & 10 & 15 & 7 & 14 & 10 & 14 & 12 & - \\
\hline \multicolumn{17}{|l|}{ Texture } \\
\hline Crunchy & 40 & 40 & 40 & 40 & 40 & 40 & 40 & 40 & 40 & 40 & 40 & 40 & 40 & 40 & 40 & 40 \\
\hline \multicolumn{17}{|l|}{ Spice flavour } \\
\hline Delightful & 40 & 40 & 40 & 40 & 40 & 40 & 40 & 40 & 40 & 40 & 40 & 40 & 40 & 40 & 40 & 40 \\
\hline \multicolumn{17}{|l|}{ Umami flavour } \\
\hline Delightful & - & 35 & 35 & 35 & - & 35 & 35 & 35 & - & 40 & 40 & 40 & & 35 & 35 & 40 \\
\hline Not perceptible & - & 5 & 5 & 5 & - & 5 & 5 & 5 & - & - & - & - & - & 5 & 5 & - \\
\hline \multicolumn{17}{|l|}{ Overall quality } \\
\hline Delicious & 27 & 28 & 31 & 30 & 20 & 30 & 32 & 31 & 20 & 35 & 30 & 30 & 20 & 20 & 30 & 30 \\
\hline Pleasant & 13 & 12 & 9 & 10 & 20 & 10 & 8 & 9 & 20 & 5 & 10 & 10 & 20 & 20 & 10 & 10 \\
\hline \multicolumn{17}{|l|}{ Lingering taste } \\
\hline Yes & 32 & 40 & 40 & 40 & 35 & 40 & 40 & 40 & 35 & 40 & 40 & 40 & 40 & 40 & 40 & 40 \\
\hline No & 8 & - & - & - & 5 & - & - & - & 5 & - & - & - & - & - & - & - \\
\hline \multicolumn{17}{|l|}{ Mouth coating } \\
\hline Yes & - & 40 & 40 & 40 & - & 40 & 40 & 40 & - & 40 & 40 & 40 & - & 40 & 40 & 40 \\
\hline No & 40 & - & - & - & 40 & - & - & & 40 & - & - & - & 40 & - & - & - \\
\hline \multicolumn{17}{|c|}{ MSG Level $2(100 \mathrm{mg} / 100 \mathrm{~g})$} \\
\hline \multicolumn{17}{|l|}{ Appearance } \\
\hline Appealing & 30 & 25 & 20 & 40 & 35 & 30 & 30 & 29 & 35 & 30 & 30 & 29 & 20 & 25 & 20 & 30 \\
\hline Attractive & 10 & 15 & 20 & & 5 & 10 & 10 & 11 & 5 & 10 & 10 & 11 & 20 & 15 & 20 & 10 \\
\hline \multicolumn{17}{|l|}{ Texture } \\
\hline Crunchy & 40 & 40 & 40 & 40 & 40 & 40 & 40 & 40 & 40 & 40 & 40 & 40 & 40 & 40 & 40 & 40 \\
\hline \multicolumn{17}{|l|}{ Spice flavour } \\
\hline Delightful & 40 & 40 & 40 & 40 & 40 & 40 & 40 & 40 & 40 & 40 & 40 & 40 & 40 & 40 & 40 & 40 \\
\hline \multicolumn{17}{|l|}{ Umami flavour } \\
\hline Delightful & - & 35 & 35 & 35 & - & 40 & 40 & 40 & - & 40 & 40 & 40 & - & 35 & 35 & 35 \\
\hline Not perceptible & - & 5 & 5 & 5 & - & - & - & - & - & - & - & - & - & 5 & 5 & 5 \\
\hline Overall quality & & & & & & & & & & & & & & & & \\
\hline Delicious & 25 & 30 & 29 & 25 & 25 & 30 & 29 & 25 & 25 & 30 & 29 & 25 & 25 & 30 & 29 & 25 \\
\hline Pleasant & 15 & 10 & 11 & 15 & 15 & 10 & 11 & 15 & 15 & 10 & 11 & 15 & 15 & 10 & 11 & 15 \\
\hline Lingering taste & & & & & & & & & & & & & & & & \\
\hline Yes & 40 & 40 & 40 & 40 & 40 & 40 & 40 & 40 & 40 & 40 & 40 & 40 & 40 & 40 & 40 & 40 \\
\hline No & - & - & - & - & - & - & - & - & - & - & - & - & - & - & - & - \\
\hline Mouth coating & & & & & & & & & & & & & & & & \\
\hline Yes & - & 40 & 40 & 40 & - & 40 & 40 & 40 & - & 40 & 40 & 40 & - & 40 & 40 & 40 \\
\hline No & 40 & - & - & - & 40 & - & - & - & 40 & - & - & - & 40 & - & - & - \\
\hline
\end{tabular}

No response for 'not appealing' under Appearance; 'brittle' and 'hard' under Texture; 'strong' and 'not perceptible' under Spice flavour; 'strong' under Umami flavour; and 'unpleasant' under Overall quality.

Table 6: Free choice profiling of poori with Mixture of spices and different levels of salt and MSG (number of responses).

to mouth coating or fatigue which sets in while dealing with strong flavours.

\section{Sensory response for products with single spice}

Low sodium poories were also prepared with single spices and different level of salt. The sensory scores given in Table 3, along with the results of statistical analysis indicate that all products prepared with chili were equally liked for the attributes of appearance, texture, spice flavour and overall quality. For umami flavour the product prepared with $1.75 \%$ salt was significantly accepted at a higher scale than others, the differences being significant. This indicated the flavor enhancing effect of MSG. In the products prepared with omum the textural quality showed a significant difference with highest score for the product with $2.0 \%$ salt and MSG. The umami flavour increased with increasing salt level though the difference was not significant. For the overall quality, there were significant differences among the products. For the products 


\begin{tabular}{|c|c|c|c|c|c|c|c|c|c|c|c|c|c|c|c|}
\hline \multirow{2}{*}{ Level of Salt (\%) } & \multicolumn{3}{|c|}{ Control Poori } & \multicolumn{3}{|c|}{ Chili } & \multicolumn{3}{|c|}{ Omum } & \multicolumn{3}{|c|}{ Cumin } & \multicolumn{3}{|c|}{ Pepper } \\
\hline & $\mathrm{L}$ & A & M & $\mathrm{L}$ & A & M & $\mathbf{L}$ & A & $M$ & $\mathbf{L}$ & A & M & $\mathbf{L}$ & A & M \\
\hline \multicolumn{16}{|l|}{ MSG (Level 1) } \\
\hline 2.0 (Con.) & - & 40 & - & - & 40 & - & - & 40 & - & - & 40 & - & - & 40 & - \\
\hline 1.5 & 15 & 25 & - & 10 & 30 & - & 10 & 30 & - & 5 & 35 & - & - & 40 & - \\
\hline 1.75 & - & 40 & - & - & 40 & - & - & 40 & - & - & 40 & - & - & 40 & - \\
\hline 2.0 & - & 30 & 10 & - & 30 & 10 & - & 25 & 15 & - & 35 & 5 & - & 40 & - \\
\hline \multicolumn{16}{|l|}{ MSG (Level 2) } \\
\hline 2.0 (Con.) & - & 40 & - & - & 40 & - & - & 40 & - & - & 40 & - & - & 40 & - \\
\hline 1.5 & 10 & 30 & - & - & 40 & - & 5 & 35 & - & - & 40 & - & - & 40 & - \\
\hline 1.75 & - & 40 & - & - & 40 & - & - & 40 & - & - & 40 & - & - & 40 & - \\
\hline 2.0 & - & 35 & 5 & - & 40 & - & - & 30 & 10 & - & 40 & - & - & 40 & - \\
\hline & & & & \multicolumn{3}{|c|}{ Chili+Omum } & \multicolumn{3}{|c|}{ Chili+Cumin } & \multicolumn{3}{|c|}{ Pepper+Omum } & \multicolumn{3}{|c|}{ Pepper+Cumin } \\
\hline \multicolumn{16}{|c|}{ MSG Level $1(75 \mathrm{mg} / 100 \mathrm{~g})$} \\
\hline 2.0 (Con.) & - & - & - & - & 40 & - & - & 40 & - & - & 40 & - & - & 40 & - \\
\hline 1.5 & - & - & - & 15 & 25 & - & 15 & 25 & - & - & 40 & - & 15 & 25 & - \\
\hline 1.75 & - & - & - & - & 40 & - & - & 40 & - & - & 40 & - & - & 40 & - \\
\hline 2.0 & - & - & - & - & 35 & 5 & - & 30 & 10 & - & 40 & - & - & 30 & 10 \\
\hline \multicolumn{16}{|c|}{ MSG Level $2(100 \mathrm{mg} / 100 \mathrm{~g})$} \\
\hline 2.0 (Con.) & - & - & - & - & 40 & - & - & 40 & - & - & 40 & - & - & 40 & - \\
\hline 1.5 & - & - & - & 10 & 30 & - & - & 40 & - & 10 & 30 & - & - & 40 & - \\
\hline 1.75 & - & - & - & - & 40 & & - & 40 & - & - & 40 & & - & 40 & - \\
\hline 2.0 & - & - & - & - & 35 & 5 & - & 40 & - & - & 35 & 5 & - & 40 & - \\
\hline
\end{tabular}

L: Low; A: Appropriate; M: More.

Table 7: Level of saltiness as felt by respondents (number of responses).

prepared with added cumin powder, appearance, texture and overall quality were similar, however the spice flavour was prominent with higher level of salt and MSG. The umami flavour also showed significant differences. The addition of pepper influenced the appearance of product with marginally significant differences. However, the flavour of spice and umami did not show any differences. The overall quality indicated that product with low level of salt were highly accepted. The overall results of this experiment indicate that spice added products can be consumed with lower sodium levels and MSG has a synergistic effect with spices.

MSG is also well known for its synergistic effect when used along with other types of ingredients. In this context an investigation was conducted to maximize umami taste of meat and to determine the acceptance level among older consumers. Products were prepared by adding tomato puree, soy sauce and soy bean paste or MSG and were compared with the control sample. A total of 7 products were developed. The observations showed that four out of 7 products had significantly higher umami contributing compounds as against control products. Products with MSG or tomato puree were found to score significantly higher for their umami/salty taste. This observation inferred that the enhanced umami or salty taste could be achieved through the incorporation of natural umami containing ingredients [24].

The descriptive quality attributes followed similar profile as earlier with high acceptability (Table 4). The free choice profiling for the above products showed uniform responses for all the qualities as observed earlier. All sensory attributes indicated that products were highly acceptable and reducing salt did not affect the taste profile adversely. The lingering taste was more prominent on addition of higher level of MSG showing the mouth coating property of MSG.

\section{Sensory response for products with mixture of spice}

The next sets of products were prepared with mixture of spices, different levels of salt and $75 \mathrm{mg}$ or $100 \mathrm{mg}$ of MSG. The sensory scores given in Table 5 and their statistical significance show a different profile than what was observed with single spices.

In this case, significant differences were observed for many sensory attributes. For chili+omum products, the appearance did not differ, however there were significant differences in texture, spice flavor and umami flavor. The products with a higher level of salt were given a higher score. The overall quality did not differ among products. In products with added chili+cumin all attributes were significantly different except for texture. While a trend was not seen with changing salt levels, the products scored highest for spice, umami flavor and overall quality with $1.75 \%$ salt. This result indicates that $12.5 \%$ reduction in salt along with MSG was optimum. The texture of product was not influenced in pepper+cumin poories, however, there were significant differences in all other attributes. Products with lesser level of salt and added MSG were accepted equally well. Here also it was seen that salt could be effectively reduced by $12.5 \%$ in MSG added products.

Apart from using mixture of spices the incorporation of mixture of umami containing ingredients could also help to improve the product acceptability. Another study on the formulation of fermented sausages with two levels of substitution of sodium chloride by potassium chloride in combination with MSG, disodium inosinate and disodium guanylate along with amino acids like lysine and taurine was reported. The investigators reported that, the replacement of sodium chloride with potassium chloride along with flavour enhancers to the extent of $50 \%$ resulted in a product having desirable physico chemical and sensory qualities [25].

The free choice profiling of these products given in Table 6 shows that all products were highly acceptable for different quality attributes. The profile was similar to other products. With mixture of spices the observations were as follows. Chili+omum influenced the textural 
quality, spice flavour and umami flavour significantly. Chili+cumin showed significant influence on spice and umami flavour. Pepper+omum altered the appearance, umami flavour and overall quality significantly whereas pepper+cumin influenced the spice and umami flavour. The free choice profiling was similar to other products. The overall results show that the mixture of spices can help in further reduction of salt wherein products with $25 \%$ less salt are also accepted well.

\section{Perception about level of saltiness}

The respondents were also asked about the level of saltiness as felt by them for each of the product. The responses are compiled in Table 7. As can be seen for the control poories some of the respondents opined that $1.5 \%$ salt was low, whereas all other levels were appropriate. The highest level of salt with MSG was felt as more by some. For chili added product at $75 \mathrm{mg}$ of MSG a similar response was seen, however, at 100 mg of MSG all products were termed as appropriate. A similar response was seen for cumin and omum. For pepper all levels were termed as appropriate. In mixture of spices, high salt and MSG was termed as more by some of the respondents. These results indicate that products were acceptable at lower level of salt; however, the differences between different levels of salt as well as added MSG were not perceptible.

\section{PCA plot}

The principal component analysis (PCA) of sensory data of the poorie samples indicated that PC1 and PC2 of MSG level $1(75 \mathrm{mg})$ accounted for 61.85 and $10.17 \%$ of total variance, and for MSG level $2(100 \mathrm{mg}$ ) products, PC1 and PC2 accounted for 60.83 and $13.49 \%$ of total variance respectively in the sensory descriptive data matrix. The PCA biplot (Figures 1A and 1B) showed that the samples were

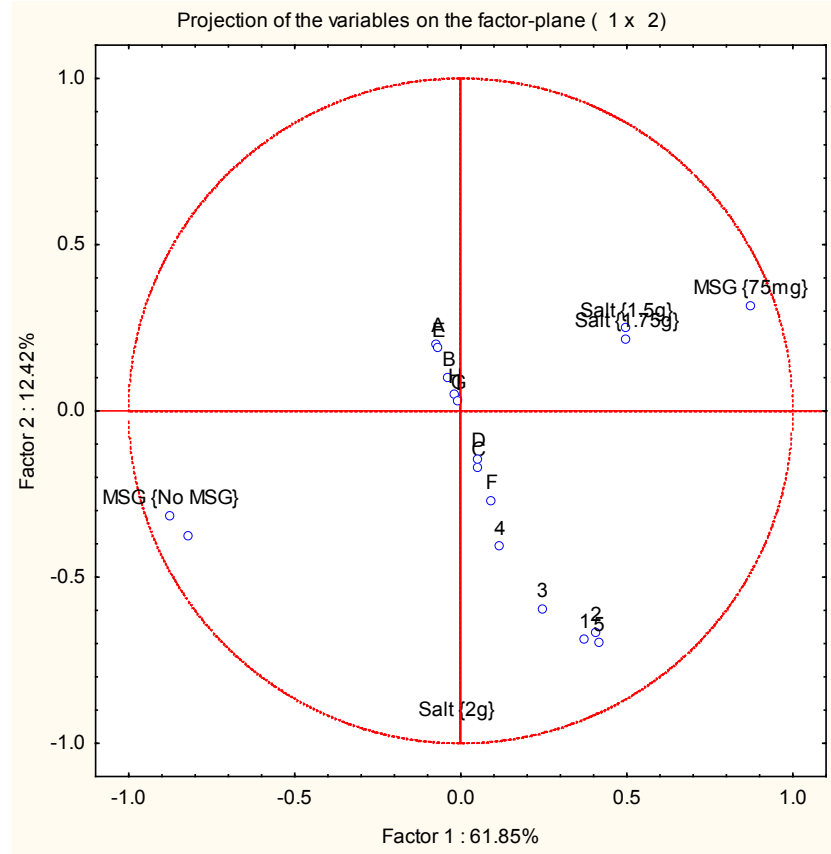

Figure 1A: Products with MSG Level 1 (75 mg/100 g). PCA plot for sensory attributes of products with two levels of MSG (Products: A. Control, B. with omum, C. with Chili, D. with Jeera, E. with pepper, F. with Chili+omum, G. with chili+cumin, H. with pepper+omum, I. pepper+cumin. Sensory Attributes: 1. Appearance; 2. Texture; 3. Perception of Spice; 4. Perception of MSG; 5. Overall Quality.

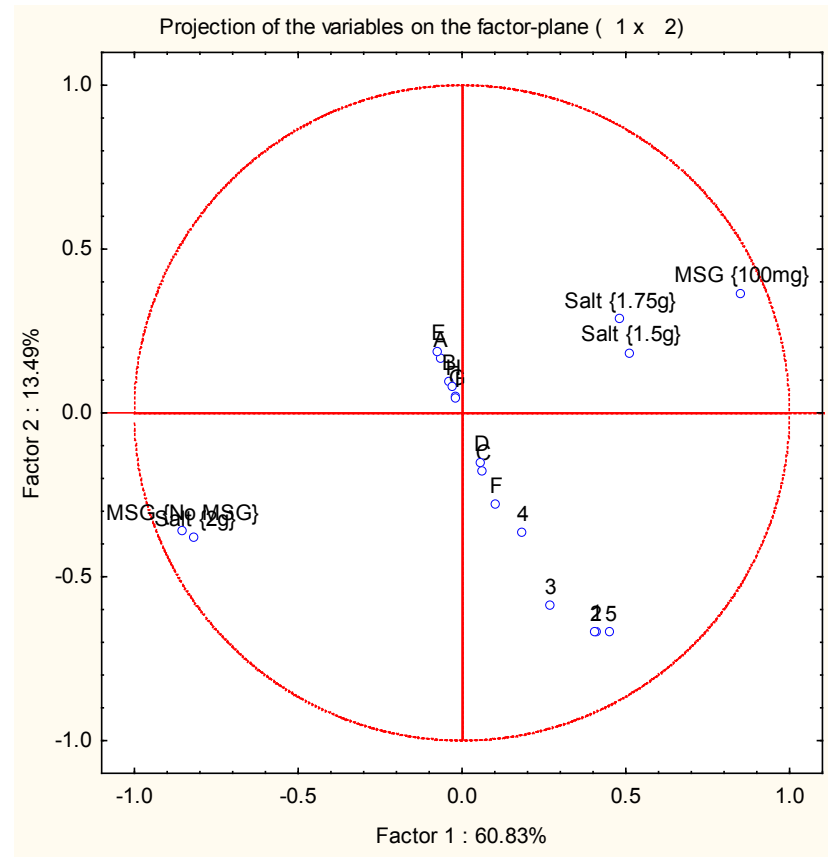

Figure 1B: Products with MSG Level $2(100 \mathrm{mg} / 100 \mathrm{~g})$. PCA plot for sensory attributes of products with two levels of MSG (Products: A. Control, B. with omum, C. with Chili, D. with Jeera, E. with pepper, F. with Chili+omum, G. with chili+cumin, H. with pepper+omum, I. pepper+cumin. Sensory Attributes: 1. Appearance; 2. Texture; 3. Perception of Spice; 4. Perception of MSG; 5. Overall Quality.

segregated according to their associated sensory descriptors. Addition of MSG improved the sensory attributes of the poories when compared with control poori. The sensory attributes of salt reduced poories demonstrated better sensory characteristics. Even with lower level of MSG addition, the salt reduction was possible in all spice combinations. MSG improved the sensory attributes of salt reduced products with all spice combinations but the chili+cumin and pepper+omum mixture were found to be the best among all spice combinations in terms of the sensory attributes and the salt reduction.

\section{Conclusion}

The present study gave certain important inferences regarding the potential benefits of substituting MSG for salt reduction along with combination of spices. The study indicated that the products with lower level of salt with added MSG were found to be well accepted. The products with least level of salt (1.5\%) along with MSG were shown to be equally accepted by all the panel members. Addition of single and mixture of spices was also found to have profound influence on the acceptability in which MSG served as a synergistic agent for improving the quality characteristics or products. Modification of salt level with different levels of MSG did not alter the quality perception as recorded by the responses of the panellists. It can be said that the MSG had a greater impact on the acceptability of the product at reduced salt levels. This addition of MSG along with spices at reduced salt levels is of particular significance for reducing salt in processed products using natural ingredients.

\section{References}

1. Bartoshu LM (1993) The biological bases of food perception and acceptance. Food Qual 4: 21-32.

2. Ikeda K (1908) On a new seasoning. J Tokyo Chem Soc 30: 820-836. 
Citation: Maheshwari HM, Prabhavathi SN, Devisetti R, Prakash J (2017) Determining Efficacy of Monosodium Glutamate for Salt Reduction in Plain and Spiced 'Poories' Through Sensory Responses. J Exp Food Chem 3: 129. doi:10.4172/2472-0542.1000129

3. Jinap S, Hajeb P (2010) Glutamate. Its applications in food and contribution to health. Appetite 55: 1-10.

4. Ninomiya K (1998) Natural occurrence. Food Rev Inter 14: 177-211.

5. Noble AC (1996) Taste-aroma interactions. Trends Food Sci Technol 7: 439444

6. Kemp SE, Beauchamp GK (1994) Flavour modification by sodium chloride and monosodium glutamate. J Food Sci 59: 682-686.

7. Marcus JB (2009) Unleashing the power of umami. Food Technol 63: 22-36.

8. Altug T, Demirag K (1993) Influence of monosodium glutamate on flavour acceptability and on the reduction of sodium chloride in some ready-made soups. Chem Chemie Mikrobiologie-Technologie der Lebensmittel 15: 161-164.

9. Chi SP, Chen TC (1992) Predicting optimum monosodium glutamate and sodium chloride concentration in chicken broth as affected by spice addition. $J$ Food Proc Preser 16: 313-326.

10. Prabhavathi SN, Prakash J (2016) Flavour potentiating effect of monosodium glutamate on acceptability profile of salt reduced tomato soups. Proceedings of $2^{\text {nd }}$ International Conference on Food Properties (iCFP), Bangkok, Thailand. 31st May-2 ${ }^{\text {nd }}$ June, 2016. ISBN-9780980825138. 1-8.

11. Kumari S, Pilerood SA, Prakash J (2014) Development and acceptability of low sodium soup mixes. Ind J Nutr Dietet 51: 65-75.

12. Maheshwari HM, Prabhavathi SN, Prakash J (2017) Exploring the flavour potentiating effect of monosodium glutamate on acceptability profile of spiced 'Poories'. Ind J Nutr Dietet 54: 265-277.

13. Prakash A, Prakash J, Prabhavathi SN (2014) Sensory attributes and shelf stability of monosodium glutamate incorporated rice crackers prepared using different oils. Ad Food Sci 36: 48-53.

14. Prabhavathi SN, Prakash J (2017) Acceptability profile of tomato soup prepared using flavor potentiator and spices. Inter J Food Nutr Dietet 5: 65-72.
15. Dahl LK (2005) Possible role of salt intake in the development of essential hypertension. Int J Epidemiol 34: 967-972.

16. De Wardener HE, He FJ, MacGregor GA (2004) Plasma sodium and hypertension. Kidney Int 66: 2454-2466.

17. Meneton P, Jeunemaitre X, De Wardener HE, MacGregor GA (2005) Link between dietary salt intakes, renal salt handling, blood pressure and cardiovascular diseases. Physiol Rev 85: 679-715.

18. Brown IJ, Tzoulaki I, Candeis V, Elliott P (2009) Salt intakes around the world, implications for public health. Int J Epidemiol 38: 791-813.

19. Vinod Raj MC, Sandeep PG, Bhavya SN, Prakash J (2015) Salt consumption among selected subjects and salt content of common processed snack foods. Ind J Nutr Dietet 52: 442-451.

20. ISI (1972) Indian standard guide for sensory evaluation of foods, Part 2 Methods and evaluation cards. Indian Standard Institution, New Delhi. 9: 28.

21. Cadello AV (1998) Perception of food quality. In Food storage stability, (Ed) Taub IA and Singh, P, CRC press, New York.

22. Mojet J, Heidema J, Christ-Hazelhof E (2004) Effect of concentration on taste-taste interactions in foods for elderly and young subjects. Chem Senses 29: 671-681.

23. Jimenez-Maroto LA, Sato T, Rankin SA (2013) Saltiness potentiation in white bread by substituting sodium chloride with a fermented soy ingredient. J Cereal Sci 58: 313-317.

24. Dos Santos BA, Campagnol PCB, Morgan MA, Pollonio MAR (2014) Monosodium glutamate, disodium inosinate, disodium guanylate, lysine and taurine improve the sensory quality of fermented cooked sausages with $50 \%$ and $75 \%$ replacement of $\mathrm{NaCl}$ with $\mathrm{KCl}$. Meat Sci 96: 509-513.

25. Dermiki M, Mounayar R, Suwankanit C, Scott J, Kennedy B, et al. (2013) Maximising umami taste in meat using natural ingredients; effect on chemistry, sensory perception and hedonic liking in young and old consumers. J Sci Food Agri 93: 3312-3321. 